\title{
¿Por qué continuamos transfundiendo dos unidades de sangre a la vez si con una sola podría ser suficiente?
}

\author{
Why do we keep transfusing two units of blood at the same time if just one could be \\ enough? \\ Por que continuamos a transfundir duas unidades de sangue ao mesmo tempo se \\ apenas uma pode ser 0 suficiente?
}

Dres. Maximiliano Berro , Ismael Rodríguez²

\section{Resumen}

La transfusión de sangre ha sido identificada como uno de los procedimientos médicos más sobreutilizados en pacientes hospitalizados. Una estrategia que permitiría reducir la exposición de los pacientes a la sangre es a través de la implementación de una política de transfusión de una sola unidad, en lugar de dos, para pacientes estables, sin hemorragia y con anemia sintomática. La decisión de transfundir sangre es compleja y depende de varios factores, además, los riesgos de la transfusión se deben sopesar contra los beneficios esperados. La transfusión de dos unidades de sangre a la vez ya no se considera una forma de tratamiento "estándar" para pacientes anémicos sin sangrado activo. Existe una gran oportunidad para reducir el uso excesivo de la transfusión y fomentar las transfusiones de una sola unidad.

Palabras clave: Transfusión sanguínea Estándares de referencia

Key words: $\quad$ Blood transfusion Reference standards

\section{Introducción}

La transfusión de sangre es un procedimiento terapéutico bien consolidado para tratar a los pacientes con anemia con más de 100 años de historia. Sin embargo, la sangre es un recurso escaso y su uso está asociado con el riesgo de efectos adversos infecciosos, inmunológicos y no inmunológicos. Ciertamente, las transfusiones pueden salvar vidas, pero a veces son innecesarias e incluso pueden ser perjudiciales. Es por esto que son continuos los esfuerzos para que la transfusión de sangre sea indicada en aquellos pacientes que se favorezcan de su relación riesgo/beneficio.

La transfusión de sangre ha sido identificada como uno de los procedimientos médicos más sobreutilizados en pacientes hospitalizados ${ }^{(1)}$. Esta determinación se basa en evidencia obtenida en forma rigurosa en ensayos clínicos prospectivos y aleatorizados, que apoyan el uso de umbrales restrictivos de hemoglobina $(\mathrm{Hb})$ para la transfusión en pacientes hemodinámicamente estables sin sangrado activo. En esos ensayos, las transfusiones fueron administradas a pacientes con umbrales de $\mathrm{Hb}$ inferiores a 7-8 g/dl (llamado umbral restrictivo) o superiores a 9-10 g/dl (llamado umbral liberal), y los resultados clínicos fueron iguales o superiores usando el umbral más bajo, lo que indica que transfundir en exceso o no es útil o incluso puede ser dañino. En estos protocolos de investigación se incorporaron políticas de transfusión de una sola unidad a la vez, y los pacientes fueron

\footnotetext{
1. Prof. Adj. Cátedra de Hemoterapia y Medicina Transfusional. Hospital de Clínicas. Facultad de Medicina. Universidad de la República. Uruguay. 2. Profesor Director Cátedra de Hemoterapia y Medicina Transfusional. Hospital de Clínicas. Facultad de Medicina. Universidad de la República. Uruguay.

Correspondencia: Dr. Maximiliano Berro. Cátedra y Departamento de Hemoterapia y Medicina Transfusional. Hospital de Clínicas "Dr. Manuel Quintela”. Av. Italia s/n, Montevideo. Uruguay. Correo electrónico: maxberro@gmail.com

Los autores del presente artículo declaran no tener conflictos de intereses.

Recibido: $10 / 6 / 20$

Aprobado: 21/10/20

Attribution-NonCommercial 4.0 International (CC BY-NC 4.0)
} 
reevaluados antes de administrarles unidades adicionales ${ }^{(2)}$.

Por lo tanto, una estrategia que permitiría reducir la exposición de los pacientes a la sangre es a través de la implementación de una política de transfusión de una sola unidad, en lugar de dos, para pacientes estables, sin hemorragia y con anemia sintomática. El concepto de que dos unidades era la dosis óptima para pacientes adultos aparece por primera vez en la década de 1960 . Surgiendo de datos obtenidos en estudios retrospectivos que exploraron principalmente el período perioperatorio. Las transfusiones de una sola unidad se asociaron con un nivel de $\mathrm{Hb}$ previo a la transfusión normal y, por lo tanto, se consideraron injustificadas con un beneficio mínimo en comparación con las transfusiones de dos unidades ${ }^{(3)}$. Estos pacientes que recibieron una sola unidad probablemente no habrían sido transfundidos según las pautas actuales. Pero en ese momento y durante muchos años la transfusión de una sola unidad fue criticada enérgicamente ${ }^{(4,5)}$. De hecho, estas creencias influyeron mucho en la práctica transfusional, incluso hasta el día de hoy, a pesar de la ausencia de evidencia que la soporte.

Cuando la transfusión de sangre está indicada, lo más apropiado es la administración de una sola unidad y luego de finalizada, la transfusión deberemos realizar una reevaluación clínica junto con el nivel de hemoglobina postransfusional, para determinar la necesidad de unidades adicionales.

Una sola unidad de sangre a menudo aliviará los síntomas de la anemia y/o elevará el nivel de hemoglobina por encima del desencadenante de la transfusión, en cuyo caso la administración de más unidades resultará innecesaria, o puede posponerse, o reemplazarse por otros tratamientos de la anemia.

\section{El umbral tansfusional restrictivo}

Existen múltiples estrategias para reducir la transfusión inapropiada de sangre. La más ampliamente aplicada y documentada es el uso de umbrales restrictivos de la transfusión. Las transfusiones de sangre fueron ampliamente adoptadas como parte del arsenal terapéutico, particularmente después de la primera y segunda guerras mundiales, sin un riguroso escrutinio científico. En ese momento, no existía lo que hoy llamamos medicina basada en la evidencia y la justificación de la transfusión parecía obvia. A partir de la década de 1980 varios factores provocaron un interés en disminuir las transfusiones. El descubrimiento de que el VIH y el virus de la hepatitis $\mathrm{C}$ podían ser transmitidos por la sangre activó alarmas sobre la seguridad de las transfusiones. Además, surgió evidencia acerca de los resultados en las cirugías llevadas a cabo en pacientes testigos de Jehová (que no aceptan transfusiones de sangre), los cuales no presentaban mayor mortalidad a pesar de presentar niveles de $\mathrm{Hb}$ muy inferiores a los que se consideraban como indicadores de la necesidad de transfusión ${ }^{(6)}$. Es así que algunos médicos comenzaron a preguntarse cuál sería el umbral transfusional correcto. Históricamente, se consideraba que la transfusión estaba indicada cuando la $\mathrm{Hb}$ se encontraba por debajo de $10 \mathrm{~g} / \mathrm{dl}$ o el hematocrito por debajo del $30 \%$, un umbral que se propuso en forma arbitraria en un artículo publicado en $1942^{(7)}$.

En 1999 se publicaron los resultados del estudio TRICC $^{(8)}$, que puso a prueba este umbral ampliamente utilizado. Los investigadores asignaron al azar a 838 pacientes de cuidados intensivos a dos grupos: en un grupo recibirían una transfusión siguiendo un umbral transfusional "liberal" si sus niveles de $\mathrm{Hb}$ eran menores de 10 g/dl, y el otro según un umbral "restrictivo" si estaban por debajo de $7 \mathrm{~g} / \mathrm{dl}$. No se encontraron diferencias significativas en la mortalidad entre ambos grupos. Además, cuando los investigadores analizaron dos subgrupos de pacientes, aquellos menores de 55 años y aquellos menos graves, descubrieron que el enfoque restrictivo en realidad había reducido la mortalidad. En los años siguientes se publicaron varios ensayos clínicos aleatorizados, cada uno de los cuales comparó el umbral restrictivo con el liberal. Se incluyeron pacientes con una amplia variedad de situaciones clínicas tales como pacientes críticos, de cirugía ortopédica, de cirugía cardíaca, pacientes con hemorragia gastrointestinal, lesiones cerebrales traumáticas y sepsis ${ }^{(9-15)}$. La conclusión fue que utilizar un umbral restrictivo no afecta su evolución e incluso en ocasiones puede favorecerlos. En estos ensayos la estrategia utilizada para la transfusión fue de una sola unidad seguida de una nueva evaluación a posteriori para determinar si el paciente requería unidades adicionales.

Actualmente disponemos de suficiente evidencia para apoyar el uso de un nivel de $\mathrm{Hb}$ inferior a 7-8 g/dl como umbral transfusional, ya que no tiene un impacto negativo en la morbimortalidad del paciente y evitaría eventos adversos. De hecho, existe unanimidad en las guías sobre indicaciones de la transfusión en este aspecto ${ }^{(16-18)}$.

\section{Los efectos adversos de la transfusión}

Las transfusiones no siempre tienen los beneficios esperados. Existen varias explicaciones posibles como, por ejemplo, que los niveles de $\mathrm{Hb}$ no son un buen indicador de la oferta de oxígeno a los tejidos, o que la sangre que están recibiendo los pacientes no se comporta igual que su propia sangre. Los glóbulos rojos frescos son flexibles y fluyen fácilmente a través de los capila- 
res más pequeños del cuerpo. Pero después de algunas semanas en un banco de sangre cambian de forma y sus membranas pierden la capacidad de deformarse, lo que provoca que obstruyan la microcirculación; además, presentan mayor afinidad por el oxígeno, lo que disminuye su oferta de éste a los tejidos hipóxicos que lo requieren. Estos cambios, conocidos como la lesión de almacenamiento, podrían hacer que los glóbulos rojos sean menos efectivos y provoquen resultados clínicos adversos. De hecho, se ha visto que existe una relación dosis dependiente entre el número de unidades transfundidas y el riesgo de mortalidad posoperatoria ${ }^{(19)}$. Cada bolsa de sangre transfundida se asocia con un incremento cada vez mayor en el riesgo de presentar resultados adversos ${ }^{(20,21)}$.

Las transfusiones pueden además transmitir enfermedades infecciosas y generar reacciones adversas, algunas de las cuales presentan una alta morbimortalidad por sí mismas. También pueden causar inmunodepresión y dejar a los pacientes más vulnerables a la infección, aunque el mecanismo detrás de este efecto aún no ha sido aclarado del todo ${ }^{(22)}$.

\section{Estudios sobre la transfusión de una versus dos unidades de sangre}

Durante años se les enseñó a los médicos que la dosis estándar de transfusión de glóbulos rojos era de dos unidades, de hecho, las transfusiones de una sola unidad eran fuertemente desaconsejadas. Ahora con numerosos ensayos clínicos que justifican el utilizar una estrategia restrictiva de transfusión, las transfusiones de sangre de una sola unidad se están convirtiendo en la práctica habitual. Hoy en día la recomendación es que en los pacientes sin sangrado activo y hemodinámicamente estables no debemos transfundir más unidades de sangre de las absolutamente necesarias ${ }^{(23)}$.

Existen varios estudios que examinaron el uso de la transfusión de una única unidad. Berger y colaboradores mostraron que no hubo diferencias en la mortalidad entre los grupos de pacientes que recibieron una sola unidad y aquellos que habían recibido dos unidades, aunque el tiempo entre episodios de transfusión se redujo un $20 \%$ en los pacientes que recibieron una en comparación con dos unidades ${ }^{(24)}$. Ma y colaboradores encontraron que adoptar una política de transfusión de una sola unidad reduce significativamente la utilización de sangre en pacientes traumatológicos, logrando el nivel de $\mathrm{Hb}$ deseado postransfusión ${ }^{(25)}$. Yang y colaboradores demostraron en una gran cohorte de pacientes que la implementación de una política de transfusión de una sola unidad tuvo un impacto mayor en la disminución de la utilización de sangre que la reducción en los umbrales transfusionales de $\mathrm{Hb}^{(2)}$. Bowman y colaboradores, del mismo modo, encontraron que cambiar la práctica de una transfusión doble a una sola unidad redujo la utilización de sangre en $29 \%$ sin comprometer la seguridad ni la eficacia ${ }^{(3)}$. Lo mismo ocurrió en el estudio de Chantepie y colaboradores, en el que notaron una reducción significativa en el número de unidades transfundidas entre pacientes con trasplante alogénico de progenitores hematopoyéticos cuando se implementó la política de transfusión de una sola unidad ${ }^{(26)}$.

En definitiva, la evidencia muestra que una política de transfusión de una sola unidad es segura, permite reducir la cantidad de transfusiones de sangre y, por lo tanto, disminuir el riesgo asociado con el uso de la sangre alogénica, sin afectar la evolución del paciente ${ }^{(27,28)}$. Para pacientes hemodinámicamente estables y en ausencia de sangrado activo, las transfusiones de sangre de una sola unidad, seguido de una reevaluación, deberían ser el estándar para la mayoría de los pacientes. La reevaluación debe incluir el nivel de $\mathrm{Hb}$ después de la transfusión y verificar la mejora en la sintomatología de la anemia o la isquemia de órganos nobles. Existe una gran oportunidad para reducir el uso excesivo de la transfusión y fomentar las transfusiones de una sola unidad ${ }^{(29)}$.

\section{¿Hay razones para indicar transfusiones de dos unidades en ciertas circunstancias?}

Sin dudas la indicación más frecuente para indicar transfusiones de múltiples unidades es el sangrado activo, ya que cualquiera que sea el umbral de $\mathrm{Hb}$ elegido, la transfusión debe administrarse en cantidades suficientes para adelantarse al sangrado, y no interrumpirse hasta que éste sea controlado. También es importante tener en cuenta el volumen intravascular, porque el aporte de oxígeno de la sangre depende de la concentración de $\mathrm{Hb}$ y del volumen sanguíneo. Si un paciente está hipovolémico y/o sangra activamente, el valor de $\mathrm{Hb}$ desencadenante de la transfusión, así como la dosis de sangre, pueden necesitar ajustarse hacia arriba, creando escenarios clínicos en los que las transfusiones de dos unidades pueden ser apropiadas ${ }^{(30)}$. Otra situación para la que se pueden indicar transfusiones de múltiples unidades incluye pacientes sintomáticos con niveles muy bajos de $\mathrm{Hb}$, en los cuales sabemos de antemano que con la transfusión de una sola unidad no podremos llegar a un nivel de $\mathrm{Hb}$ seguro. Otros pacientes que pueden beneficiarse de niveles más altos de $\mathrm{Hb}$ logrados por transfusiones de dos unidades incluyen aquellos con anemia crónica y que son dependientes de la transfusión. La transfusión de una sola unidad disminuye el tiempo entre transfusiones en este tipo de pacientes, por lo que muchas veces es preferible realizar transfusiones de dos unidades para optimizar el tratamiento y mejorar la calidad de vida del paciente. 
Es importante recordar que tratamos a los pacientes y sus síntomas, no solo sus valores de laboratorio. La buena atención médica adapta o modifica los protocolos y pautas de tratamiento de acuerdo con la situación clínica. Por lo que en muchos casos puede estar indicada la transfusión de dos unidades a la vez. Lo importante es que esta indicación se base en un análisis completo y no que resulte de un acto reflejo.

\section{Conclusiones}

La transfusión de sangre tiene una posición e importancia irremplazables en el tratamiento de pacientes con anemia y sangrado. Debemos saber que la sangre es como cualquier otro agente terapéutico: no está exento de riesgos, tiene un costo y debe administrarse solo cuando esté indicada y con la dosis efectiva más baja. Por esto es imprescindible tener un buen dominio de las indicaciones de la transfusión en la práctica clínica.

La decisión de transfundir sangre es compleja y depende de varios factores, como ser la causa de la anemia, su severidad y tiempo de evolución, la capacidad del paciente para compensarla, la probabilidad del sangrado y la necesidad de mantener una reserva antes de la instalación de hipoxia tisular. Los riesgos de la transfusión también se deben sopesar contra los beneficios esperados. Aunque las guías establecen umbrales de $\mathrm{Hb}$ para orientar la transfusión con el objetivo de ser pragmáticas, la correcta evaluación del cuadro clínico del paciente es lo más importante al tomar la decisión de transfundir. La transfusión de dos unidades de sangre a la vez ya no se considera una forma de tratamiento "estándar" para pacientes anémicos sin sangrado activo. Aunque cambiar esta práctica resulta difícil, modificar décadas de práctica médica establecida no es fácil. Incluso cuando las pautas son claras, a menudo los médicos no las siguen. Nadie que examine objetivamente la evidencia puede estar en desacuerdo en que debemos reducir la exposición a la transfusión en nuestros pacientes tanto como sea posible. Es por esto que debemos continuar los esfuerzos para que las tasas de transfusión se reduzcan, a través de la práctica de la transfusión de una sola unidad, que reduce el riesgo potencial de la transfusión alogénica con el beneficio que esto representa para los pacientes.

\section{Summary}

Blood transfusion has been identified as one of the most overused medical procedures in hospitalized patients. One strategy that would reduce the exposure of patients to blood is through the implementation of a single-unit regime for stable patients without bleeding and with symptomatic anemia. The decision to transfuse blood is complex and depends on several factors, in addition, the risks of transfusion must be weighed against the expected benefits. Transfusion of two units of blood at the same time is no longer considered a "standard" form of treatment for anemic patients without active bleeding. There is a great opportunity to reduce the overuse of transfusion and encourage single-unit transfusions.

\section{Resumo}

A transfusão de sangue foi identificada como um dos procedimentos médicos usados de mais em pacientes hospitalizados. Uma estratégia que reduziria a exposição dos pacientes ao sangue é por meio da implantação de uma política transfusional de unidade única para pacientes estáveis, sem sangramento e com anemia sintomática. A decisão de transfundir sangue é complexa e depende de vários fatores, além disso, os riscos da transfusão devem ser analisados em relação aos benefícios esperados. A transfusão de duas unidades de sangue ao mesmo tempo não é mais considerada uma forma "padrão" de tratamento para pacientes anêmicos sem sangramento ativo. Há uma grande oportunidade de reduzir o uso excessivo de transfusões e incentivar as transfusões de uma única unidade.

\section{Bibliografía}

1. Goodnough L, Maggio P, Hadhazy E, Shieh L, Hernández-Boussard T, Khari P, et al. Restrictive blood transfusion practices are associated with improved patient outcomes. Transfusion 2014; 54(10 Pt 2):2753-9. doi: 10.1111/trf. 12723

2. Yang W, Thakkar R, Gehrie E, Chen W, Frank S. Single-unit transfusions and hemoglobin trigger: relative impact on red cell utilization. Transfusion 2017; 57(5):1163-70. doi: $10.1111 /$ trf. 14000

3. Bowman Z, Fei N, Ahn J, Wen S, Cumpston A, Shah N, et al. Single versus double-unit transfusion: Safety and efficacy for patients with hematologic malignancies. Eur J Haematol 2019; 102(5):383-8. doi: 10.1111/ejh.13211

4. Cass R, Blumberg N. Single-unit blood transfusion: doubtful dogma defeated. JAMA 1987; 257(5):628-9.

5. Mintz P. Single-unit blood transfusions. N Engl J Med 1983; 309(10):614. doi: 10.1056/nejm198309083091015

6. Thomson A, Farmer S, Hofmann A, Isbister J, Shander A. Patient blood management - a new paradigm for transfusion medicine? ISBT Sci Ser 2009; 4:423-35. doi: 10.1111/j.1751-2824.2009.01251.x

7. Adams R, Lundy J. Anesthesia in cases of poor surgical risk: some suggestions for decreasing the risk. Surgery, Gynecology \& Obstetrics. 1942; 74:1011-19.

8. Hébert P, Wells G, Blajchman M, Marshall J, Martin C, Pagliarello G, et al. A multicenter, randomized, controlled clinical trial of transfusion requirements in critical care. Transfusion Requirements in Critical Care Investigators, Ca- 
nadian Critical Care Trials Group. N Engl J Med 1999; 340(6):409-17. doi: 10.1056/NEJM199902113400601

9. Lacroix J, Hébert P, Hutchison J, Hume H, Tucci M, Ducruet $\mathbf{T}$, et al. Transfusion strategies for patients in pediatric intensive care units. N Engl J Med 2007; 356(16):1609-19. doi: 10.1056/NEJMoa066240

10. Hajjar L, Vincent J, Galas F, Nakamura R, Silva C, Santos $\mathrm{M}$, et al. Transfusion requirements after cardiac surgery: the TRACS randomized controlled trial. JAMA 2010; 304(14):1559-67. doi: 10.1001/jama.2010.1446

11. Carson J, Terrin M, Noveck H, Sanders D, Chaitman B, Rhoads G, et al. Liberal or restrictive transfusion in high-risk patients after hip surgery. N Engl J Med 2011; 365(26):2453-62. doi: 10.1056/NEJMoa1012452

12. Villanueva C, Colomo A, Bosch A. Transfusion for acute upper gastrointestinal bleeding. N Engl J Med 2013; 368(14):1362-3. doi: 10.1056/NEJMc1301256

13. Robertson C, Hannay H, Yamal J, Gopinath S, Goodman J, Tilley B. Effect of erythropoietin and transfusion threshold on neurological recovery after traumatic brain injury: a randomized clinical trial. JAMA 2014; 312(1):36-47. doi: 10.1001/jama.2014.6490

14. Holst L, Haase N, Wetterslev J, Wernerman J, Guttormsen A, Karlsson S, et al. Lower versus higher hemoglobin threshold for transfusion in septic shock. N Engl J Med 2014; 371(15):1381-91. doi: 10.1056/NEJMoa1406617

15. Mazer C, Whitlock R, Fergusson D, Hall J, Belley-Cote E, Connolly K, et al. Restrictive or liberal red-cell transfusion for cardiac surgery. N Engl J Med 2017; 377(22):2133-44. doi: 10.1056/NEJMoa1711818

16. Carson J, Guyatt G, Heddle N, Grossman B, Cohn C, Fung M, et al. Clinical practice guidelines from the AABB: red blood cell transfusion thresholds and storage. JAMA 2016; 316(19):2025-35. doi: 10.1001/jama.2016.9185

17. Mueller M, Van Remoortel H, Meybohm P, Aranko K, Aubron C, Burger R, et al. Patient blood management: recommendations from the 2018 Frankfurt Consensus Conference. JAMA 2019; 321(10):983-97. doi: 10.1001/jama.2019.0554

18. Retter A, Wyncoll D, Pearse R, Carson D, McKechnie S, Stanworth S, et al. Guidelines on the management of anaemia and red cell transfusion in adult critically ill patients. Br J Haematol 2013; 160(4):445-64. doi: 10.1111/bjh.12143

19. Ferraris V, Davenport D, Saha S, Austin P, Zwischenberger J. Surgical outcomes and transfusion of minimal amounts of blood in the operating room. Arch Surg 2012; 147(1):49-55. doi: 10.1001/archsurg.2011.790

20. Koch C, Li L, Duncan A, Mihaljevic T, Cosgrove D, Loop F, et al. Morbidity and mortality risk associated with red blood cell and blood-component transfusion in isolated coronary artery bypass grafting. Crit Care Med 2006; 34(6):1608-16. doi: 10.1097/01.CCM.0000217920.48559.D8

21. Santos A, Sousa A, Piotto R, Pedroso J. Mortality risk is dose-dependent on the number of packed red blood cell transfused after coronary artery bypass graft. Rev Bras Cir Cardiovasc 2013; 28(4):509-17. doi: 10.5935/1678-9741.20130083

22. Anthes E. Evidence-based medicine: Save blood, save lives. Nature 2015; 520(7545):24-6. doi: 10.1038/520024a

23. Podlasek S, Thakkar R, Rotello L, Fleury T, Demski R, Ness $\mathbf{P}$, et al. Implementing a "Why give 2 when 1 will do?" Choosing Wisely campaign. Transfusion 2016; 56(9):2164. doi: $10.1111 /$ trf. 13664

24. Berger M, Gerber B, Arn K, Senn O, Schanz U, Stussi G. Significant reduction of red blood cell transfusion requirements by changing from a double-unit to a single-unit transfusion policy in patients receiving intensive chemotherapy or stem cell transplantation. Haematologica 2012; 97(1):116-22. doi: 10.3324/haematol.2011.047035

25. Ma M, Eckert K, Ralley F, Chin-Yee I. A retrospective study evaluating single-unit red blood cell transfusions in reducing allogeneic blood exposure. Transfus Med 2005; 15(4):307-12. doi: 10.1111/j.0958-7578.2005.00592.x

26. Chantepie S, Mear J, Parienti J, Bazin A, Benabed K, Cheze S, et al. Blood transfusion in hematologic intensive care unit. Transfusion 2017; 57(2):296-302. doi: 10.1111/trf. 13908

27. Carson J, Carless P, Hebert P. Transfusion thresholds and other strategies for guiding allogeneic red blood cell transfusion. Cochrane Database Syst Rev 2012; 4(4):CD002042. doi: 10.1002/14651858.CD002042.pub3

28. Heyes J, Kelly P, Monaghan K, Lawn M, Dhesi A, Mijovic A. A single unit transfusion policy reduces red cell transfusions in general medical in-patients. QJM 2017; 110(11):735-9. doi: 10.1093/qjmed/hcx150

29. Shih A, Liu A, Elsharawi R, Crowther M, Cook R, Heddle N. Systematic reviews of guidelines and studies for single versus multiple unit transfusion strategies. Transfusion 2018; 58(12):2841-60. doi: 10.1111/trf.14952

30. Thakkar R, Podlasek S, Rotello L, Ness P, Frank S. Two-unit red cell transfusions in stable anemic patients. J Hosp Med 2017; 12(9):747-9. doi: 10.12788/jhm.2806

\section{Contribución de autores}

Ambos autores participaron en igual medida en las distintas etapas de elaboración del artículo.

Maximiliano Berro, https://orcid.org/0000-0002-7513-5466

Ismael Rodríguez, https://orcid.org/0000-0002-3361-7117 